\title{
BIRELATIONS AND SIDON SETS ${ }^{1}$
}

\author{
S. W. DRURY
}

ABSTRACT. It is shown that a knowledge of the set of birelations with coefficients $-1,0,1$ supported on a subset of a discrete abelian group is enough to determine whether the subset has the Sidon property or not.

1. Statement of the result. Let $X$ be a subset of a discrete abelian group $G$. We say that $X$ is Sidon if every bounded complex-valued function on $X$ is the restriction to $X$ of a Fourier-Stieltjes transform on $G$. We refer the reader to [1] for the elementary theory of Sidon sets, unexplained notations and definitions.

Given complete knowledge of the group of relations on $X$ it is possible in theory to determine whether $X$ is Sidon or not. How to do this "in practice' is an open question. The object of this note is, roughly speaking, to show that complete knowledge of the set of relations on $X$ with coefficients $-1,0,1$ is in theory enough to decide whether or not $X$ is Sidon. It should be noted that many of the classical combinatorial arithmetic conditions sufficient for $X$ to be Sidon depend only on relations with small coefficients [1], [3]. .

In fact the concept of a relation is a bad one in this context since only certain relations count-the birelations. We view a birelation as a function $n: X \rightarrow \mathbf{Z}$ of finite support such that

$$
\sum_{x \in X} n(x) \cdot x=O_{G}, \quad \sum_{x \in X} n(x)=O_{Z} .
$$

Indeed we view the group of all functions $n: X \rightarrow \mathrm{Z}$ of finite support (under pointwise operations) as the free abelian group generated by $X$. The canonical embedding $j: X \rightarrow$ free $X$ is given by

$$
\begin{array}{rlrl}
(j(x))(y)=1 & & \text { if } x=y, \\
& =0 & & \text { if } x \neq y .
\end{array}
$$

We denote by $Q_{N}$ the subset of free $X$ given by $\{n ; n \in$ free $(X),|n(x)| \leq$ $N \forall x \in X\}$.

Let $X$ be an abstract set embedded in discrete abelian groups $G_{k}$ $(k=1,2)$ in two (different) ways. Let $R_{k}$ be the corresponding groups of birelations in free $X$.

Received by the editors August 26, 1974 .

AMS (MOS) subject classifications (1970). Primary 43A25.

Key words and phrases. Sidon set, Fourier-Stieltjes transform, relation subgroup. of Canada.

1 The research for this article was supported by the National Research Council 
Theorem. If $R_{1} \cap Q_{1}=R_{2} \cap Q_{1}$ then either $X$ is Sidon in both embed. dings or $X$ is non-Sidon in both embeddings.

2. Reduction to finite sets $X$. If $X$ is Sidon then $X$ is Sidon $(a)$ for some $a(0<a \leq 1)$. Here Sidon $(\alpha)$ is characterized by one of the following equivalent definitions.

$$
\begin{gathered}
\alpha\|\phi\|_{B(X)} \leq\|\phi\|_{C(X)} \quad \forall \phi \in C(X), \\
\|\lambda\|_{P . M} \geq \alpha\|\lambda\|_{M} \quad \forall \lambda \in M(X) .
\end{gathered}
$$

To prove the Theorem it suffices to show that if $R_{1} \cap Q_{1}=R_{2} \cap Q_{1}$ and $X$ is Sidon in $G_{1}$, then $X$ is Sidon in $G_{2}$. Thus, by reason of the fact that $X$ is Sidon $(\alpha)$ if and only if all its finite subsets are, we need only show the following in the case $N=1$.

Proposition. $\forall \alpha(0<\alpha \leq 1), \forall N \geq 1 \exists \beta=\beta(\alpha, N)$ with $0<\beta \leq 1$ such that if $X$ is finite Sidon $(\alpha)$ in $G_{1}$ and $R_{1} \cap Q_{N}=R_{2} \cap Q_{N}$ then $X$ is Sidon $(\beta)$ in $G_{2}$. (Actually one may show $\beta \geq \alpha(1-1 / N)$ if $N \geq 2$.)

3. A new embedding. The compact dual group of free $X$, denoted $\Omega$, is identified to the group of unimodular functions on $X$ under pointwise multiplication and convergence, the duality being given by

$$
\langle\omega, n\rangle=\prod_{x \in X} \omega(x)^{n(x)} .
$$

The annihilator of the birelations subgroup $R$ in $\Omega$ is denoted $\Gamma$ and identified to

$$
\Gamma=\left\{\left.z \cdot \chi\right|_{X} ; z \in \mathbf{C},|z|=1, \chi \in \hat{G}\right\}
$$

We may evaluate the elements of $\Gamma$ at points of $X$ and these evaluations are distinct. Thus $X$ may be embedded as a subset of $\hat{\Gamma}$. This embedding has the same birelations as the original embedding. It has no relations which are not birelations. In particular $O_{\hat{\boldsymbol{\Gamma}}}$ does not lie in $X$.

The following lemma is an immediate consequence of criterion (3).

Lemma 1. If $X$ is Sidon $(\beta)$ in $\hat{\Gamma}$ then $X$ is Sidon $(\beta)$ in $G$.

In the opposite direction we have the following more technical result.

Lemma 2. Let $X$ be a finite Sidon $(\alpha)$ subset of $G$ and let $\epsilon>0$. Then there exist positive measures $\mu_{\omega}$ on $\Gamma$ such that
(a) $\left\|\mu_{\omega}\right\|_{M}=(1+\epsilon) \alpha^{-1} \forall \omega \in \Omega$,
(b) $\hat{\mu}_{\omega}(x)=\omega(x) \forall x \in X \forall \omega \in \Omega$,
(c) $\omega \rightarrow \mu_{\omega}$ is norm continuous on $\Omega$. 
Here (b) is to be interpreted relative to the new embedding. As there is little new in this lemma we merely sketch the proof.

Firstly let $\operatorname{co}(\Omega)$ denote the convex hull of $\Omega$ that is the set of maps of $X$ into the closed unit disc. For each $\phi \in \operatorname{co}(\Omega)$ we show $\exists \mu_{\phi} \in M^{+}(\Gamma)$ such that

$$
\begin{aligned}
& \left(\mathrm{a}_{1}\right)\left\|\mu_{\phi}\right\|_{M} \leq(1+\epsilon) \alpha^{-1}, \\
& \left(\mathrm{~b}_{1}\right) \hat{\mu}_{\phi}(x)=\phi(x) \forall x \in X, \\
& \text { Let } C=\left\{\left.\hat{\mu}\right|_{X} ; \mu \in M^{+}(\Gamma) \text { satisfies }\left(\mathrm{a}_{1}\right)\right\} \text {, a closed convex subset of }
\end{aligned}
$$
$C(X)$. It remains to show that $\phi \in C$. If not, by Hahn-Banach $\exists \lambda \in M(X)$ such that

$$
\begin{gathered}
R\langle\lambda, \phi\rangle \geq 1, \\
R\langle\lambda, u\rangle \leq 1 \quad \forall u \in C .
\end{gathered}
$$

But (4) implies that $\|\lambda\|_{M} \geq 1$ and (5) that $(1+\epsilon)\|\lambda\|_{P M} \leq \alpha$ which together contradict (3).

Secondly we show that for each $\phi \in \operatorname{co}(\Omega), \exists \mu_{\phi} \in M^{+}(\Gamma)$ such that $\left(a_{1}\right)$,

$$
\left|\hat{\mu}_{\phi}(x)-\phi(x)\right| \leq \epsilon \forall x \in X,
$$

hold. Indeed this follows from a routine 'partition of unity' argument on $\operatorname{co}(\Omega)$.

Thirdly we show that for each $\phi \in \operatorname{co}(\Omega){ }^{\exists} \mu_{\phi} \in M^{+}(\Gamma)$ such that $\left(\mathrm{a}_{1}\right)$, $\left(b_{1}\right)$ and $\left(c_{2}\right)$ hold. This follows from a routine iteration argument (which involves a change in $\epsilon$ ).

Fourthly we show that for each $\phi \in \operatorname{co}(\Omega) \exists \mu_{\phi} \in M^{+}(\Gamma)$ such that $\left\|\mu_{\phi}\right\|_{M}$ $=(1+\epsilon) a^{-1},\left(b_{1}\right)$ and $\left(c_{2}\right)$ hold. This follows by adding in the measure $\left((1+\epsilon) \alpha^{-1}-\left\|\mu_{\phi}\right\|_{M}\right) \eta_{\Gamma}$, where $\eta_{\Gamma}$ denotes the Haar measure on $\Gamma$. Since $O_{\hat{\mathbf{\Gamma}}}$ does not lie in $X$ as embedded in $\hat{\Gamma}$, this does not affect the value of $\hat{\mu}_{\phi}$ on $X$.

Finally restricting the choice of $\phi$ to $\Omega$ gives the required result.

4. Proof of Proposition 1 in the case $N=2$. We deal with this case first as it is the easiest to understand.

Let $\epsilon>0$ be arbitrary. For $\rho \in \Omega$ let $\mu_{\rho}$ denote the measure of Lemma 2 applied to the embedding $X \subseteq G_{1}$. We define for $\omega \in \Omega$ and $\chi \in \Gamma_{2}$ the integrated Riesz product

$$
R(\omega, \chi)=\iint \prod_{x \in X}\left(1+R\left(\omega \rho^{-1} \chi \phi^{-1}\right)(x)\right) d \mu_{\rho}(\phi) d \eta_{\Omega}(\rho)
$$

where $\eta_{\Omega}$ is Haar measure on $\Omega$ and $\phi$ denotes a generic element of $\Gamma_{1}$. Clearly $R$ is a nonnegative continuous function on $\Omega \times \Gamma_{2}$. Thus $\|R(\omega, \cdot)\|_{1}$ $=\hat{R}\left(\omega, O_{\hat{\boldsymbol{\Gamma}}_{2}}\right)$ and a calculation shows that this equals 


$$
\sum \iint\left(\frac{1}{2}\right)^{\Sigma|n(x)|}\left\langle\omega \rho^{-1} \phi^{-1}, n\right\rangle d \mu_{\rho}(\phi) d \eta_{\Omega}(\rho)
$$

where the inner sum is taken over $x \in X$ and the outer one over $n \in Q_{1} \cap$ $R_{2}$. Since by hypothesis $Q_{1} \cap R_{2}=Q_{1} \cap R_{1} \subseteq R_{1}$, we have $\left\langle\phi^{-1}, n\right\rangle=1$ in each term. The integrand with respect to $\mu_{\rho}$ is then constant and by (a) we have

$$
\|R(\omega, \cdot)\|_{1}=(1+\epsilon) \alpha^{-1} \sum \int\left(\frac{1}{2}\right)^{\Sigma|n(x)|}\left\langle\omega \rho^{-1}, n\right\rangle d \eta_{\Omega}(\rho) .
$$

On integration by $\eta_{\Omega}$ the only term to give a contribution is the one corresponding to $n=0$. Hence

$$
\|R(\omega, \cdot)\|_{1}=(1+\epsilon) \alpha^{-1} \quad \forall \omega \epsilon \Omega .
$$

A similar calculation shows that for $y \in X, \hat{R}(\omega, y)$ equals (6) where the outer sum is now taken over $\left\{n ; n \in Q_{1}, n-j(y) \in R_{2}\right\}$, For such $n, n-j(y)$ $\epsilon Q_{2} \cap R_{2}=Q_{2} \cap R_{1} \subseteq R_{1}$ so that in each term $\left\langle\phi^{-1}, n\right\rangle=\phi^{-1}(y)$. Using (b) we obtain

$$
\hat{R}(\omega, y)=\sum \int\left(\frac{1}{2}\right)^{\Sigma|n(x)|} \rho(y)\left\langle\omega \rho^{-1}, n\right\rangle d \eta_{\Omega}(\rho)=\frac{1}{2} \omega(y) .
$$

It follows that $X$ is Sidon $(1 / 2 \alpha)$ in $\hat{\Gamma}_{2}$ and hence also in $G_{2}$ by Lemma 1 .

The particular estimates $\beta=(1-1 / N) \alpha$ for general $N \geq 2$ are obtained by a similar argument applied to the corresponding Fejer kernel Riesz product

$$
R(\omega, \chi)=\iint \prod_{x \in X} K_{N}\left(\left(\omega \rho^{-1} \chi \phi^{-1}\right)(x)\right) d \mu \rho(\phi) d \eta_{\Omega}(\rho)
$$

where $K_{N}(z)=\Sigma_{|n| \leq N}(1-|n| / N) z^{n}$ denotes the Fejer kernel.

5. Proof of Proposition 1 in case $N=1$. In this section we extend the proof of $\$ 4$ by adapting two well-known ideas. The following variant of Lem. ma 2 is based on Drury $[2, \$ 2]$.

Lemma 3. Let $X$ be a finite Sidon $(\alpha)$ subset of $G$ and let $\epsilon>0$. Then there exist positive measures $\nu_{\omega}$ on $\Gamma$ such that

(a') $\left\|\nu_{\omega}\right\|_{M}=(1+\epsilon) \alpha^{-2} \forall \omega \in \Omega$,

(b') $\hat{\nu}_{\omega}(x)=\omega(x) \forall x \in X \forall \omega \in \Omega$,

(c') $\omega \rightarrow \nu_{\omega}$ is norm continuous on $\Omega$,

(d') $\int\langle\omega, n\rangle \hat{\nu}_{\omega}(u) d \eta_{\Omega}(\omega) \geq 0 \forall u \in \hat{\Gamma} \forall n \in$ free $X$.

Proof. Let $\mu_{\omega}$ be the measure of Lemma 2 and set $\nu_{\omega}=\int \mu_{\omega \lambda}-1 *$ $\tilde{\mu}_{\lambda} d \eta_{\Omega}(\lambda)$ where $\sim$ denotes the usual involution on $M(\Gamma)$ and $\lambda$ is a generic element of $\Omega$. After suitable juggling with $\epsilon$, the first three conditions are 
easily verified. Condition ( $\left.d^{\prime}\right)$ follows since

$$
\int\langle\omega, n\rangle \hat{\nu}_{\omega}(u) d \eta_{\Omega}(\omega)=\left|\int\langle\omega, n\rangle \hat{\mu}_{\omega}(u) d \eta_{\Omega}(\omega)\right|^{2} \geq 0 .
$$

This completes the proof of Lemma 3.

With $\epsilon>0,0<\delta \leq 1$ and the $\nu_{\rho}$ of Lemma 3, we define

$$
R_{\delta}(\omega, \chi)=\iint \prod_{x \in X}\left(1+\delta R\left(\omega \rho^{-1} \chi \phi^{-1}\right)(x)\right) d \nu_{\rho}(\phi) d \eta_{\Omega}(\rho) .
$$

As in $\$ 4, R_{\delta}$ is a nonnegative continuous function on $\Omega \times \Gamma_{2}$ satisfying

$$
\left\|R_{\delta}(\omega, \cdot)\right\|_{1}=(1+\epsilon) \alpha^{-2} \quad \forall \omega \in \Omega .
$$

In particular this holds for $\omega=1$ and $\delta=1$. Thus

$$
\left|\hat{R}_{1}(1, y)\right| \leq(1+\epsilon) \alpha^{-2} \quad \forall y \in X \subseteq \hat{\Gamma}_{2} .
$$

The use of (8) in the control of $\hat{R}_{\delta}(\omega, y)$ stems from an idea of Rider [3, Lemma 1.7]. We have

$$
\hat{R}_{\delta}(\omega, y)=\sum\left(\frac{\delta}{2}\right)^{\Sigma|n(x)|} a(n)\langle\omega, n\rangle
$$

where the inner summation is over $x \in X$, the outer sum is over

$$
S=\left\{n ; n \in Q_{1}, n-j(y) \in R_{2}\right\}
$$

and

$$
a(n)=\iint\left\langle\rho^{-1} \phi^{-1}, n\right\rangle d \eta_{\rho}(\phi) d \eta_{\Omega}(\rho) .
$$

As in $\$ 4$ we have $a(j(y))=1$. Thus by (9),

$$
\left|\hat{R}_{\delta}(\omega, y)-1 / 2 \delta \omega(y)\right| \leq \sum\left(\frac{\delta}{2}\right)^{\Sigma|n(x)|}|a(n)|
$$

where the outer sum is now over $S \backslash\{j(y)\}$. By (1), if $n \in S$, then $\Sigma|n(x)|$ is odd. Since the points of $X$ are distinct (in $G_{2}$ ) it follows that $\Sigma|n(x)|$ $\geq 3 \forall n \in S \backslash\{j(y)\}$. Thus by (10), and since $\delta \leq 1$, we have

$$
\left|\hat{R}_{\delta}(\omega, y)-1 / 2 \delta \omega(y)\right| \leq \delta^{3} \sum\left(\frac{1}{2}\right)^{\Sigma|n(x)|}|a(n)|,
$$

the outer sum still being taken over $S \backslash\{j(y)\}$. By $\left(\mathrm{d}^{\prime}\right), a(n)$ is always nonnegative. Together with (8) and (9) this shows that

$$
\sum_{S}\left(\frac{1}{2}\right)^{\Sigma|n(x)|}|a(n)| \leq(1+\epsilon) \alpha^{-2} .
$$

Using this estimate in (11) we have

$$
\left|\hat{R}_{\delta}(\omega, y)-1 / 2 \delta \omega(y)\right| \leq \delta^{3}\left((1+\epsilon) \alpha^{-2}-1 / 2\right) .
$$

This together with (7) and the standard iteration argument shows that $X$ is 
Sidon $(\beta)$ in $\hat{\Gamma}_{2}$ and hence also in $G_{2}$. For an arbitrarily small value of $\epsilon$ and $\delta=3^{-1 / 2} \alpha\left(2-\alpha^{2}\right)^{-1 / 2}$ we may take $\beta$ to be $3^{-3 / 2} \alpha^{3}\left(2-a^{2}\right)^{-1 / 2}$.

\section{REFERENCES}

1. W. Rudin, Fourier analysis on groups, Interscience Tracts in Pure and Appl. Math., no. 12, Interscience, New York, 1962. MR 27 \#2808.

2. S. W. Drury, Unions of sets of interpolation, Conf. on Harmonic Analysis, University of Maryland, Lecture Notes in Math., no. 266, Springer-Verlag, New York, 1972.

3. D. G. Rider, Gap series on groups and spheres, Canad. J. Math. 18 (1966), 389398. MR $32 \# 8047$.

DEP ARTMENT OF MATHEM ATICS, McGILL UNIVERSITY, MONTREAL 101, CANADA 